\title{
Community Public Art Design Practice from the Perspective of Ecological Aesthetics - The Example of Railway Three Village in Chongqing
}

\author{
Sun Lei ${ }^{1, a}$, Fan Xiaoli ${ }^{2, b^{*}}$ \\ ${ }^{I}$ School of Design, Jiangnan University, Wuxi, Jiangsu, China \\ ${ }^{2}$ School of Design, Jiangnan University, Wuxi, Jiangsu, China \\ a810405129@qq.com \\ b*85953843@qq.com

\section{ABSTRACT} \\ This paper analyses the creation process and the advantages of the intervention of the community public art work The \\ Hall of All Souls in Chongqing's Railway Three Villages, and integrates the concept of ecological aesthetics with the \\ principles of in-terrain, public, participatory and ecological community public art and launches a practical creation, with \\ a view to providing ideas for community public art design practice in community spaces from an ecological aesthetic \\ perspective.
}

Keywords: Public Art, Community Public Art, Ecological Aesthetics

\section{生态美学视角下的社区公共艺术设计实践一一以重庆 市铁路三村为例}

孙否 $1, \mathrm{a}$ 范晓莉 $2, \mathrm{~b}^{*}$

1 江南大学设计学院, 无锡, 江苏, 中国

2 江南大学设计学院, 无锡, 江苏, 中国

a810405129@qq.com

b*85953843@qq.com

摘要

社区公共艺术作为城市社区更新的重要手段之一，在实践中起到了美化社区空间，促进邻里关系的作用，本文 分析了重庆市铁路三村中社区公共艺术作品《众生堂》的创作过程，和介入优势，并将生态美学的概念与社区 公共艺术的在地形、公众性、参与性、生态性的原则相融合并展开实践创作，以期为社区空间中生态美学视角 下的社区公共艺术设计实践提供思路。

关键词: 公共艺术，社区公共艺术，生态美学

\section{1. 生态美学和社区公共艺术}

\section{1. 生态美学}

生态美学即为生态学与美学融合而成的一门学 科, 生态学是主要研究生物及其生存环境关系的学 科, 而美学则是探索人与现实审美功能性的学科, 这
两门学科在探索人与自然、人与环境相互关系上找到 了平衡点, 即为生态美学。对于生态美学目前学术上 有狭义与广义两种解释。狭义的生态美学可以理解为 着眼于人与自然环境的生态审美关系，提出特殊的 生态美范畴。而广义的生态美学可以理解为包括人与 自然、社会以及自身的生态审美关系，是一种符合生 态规律的存在论美学观。总体来说生态美学是人与社 
会环境和谐相处的状态下的崭新的生态存在下的论 美学观念。

\section{2. 社区公共艺术}

随着城市存量更新的发展, 公共艺术也随之从城 市空间逐渐转移到社区公共空间，主题逐渐由艺术家 转向了社区公民。不同于以往的城市公共艺术, 社区 中的公共艺术更强调围绕社群话题展开创作，注重居 民的利益, 主张强调居民在公共艺术实践中发挥的作 用。这一理念与美国艺术家苏珊提出的 “新类型公共 艺术” (New Genre Public art), 也被称为 “社区艺术”

(Community art) 的概念相似: 以公众议题为导向, 以公共利益为出发点、以社群为基础、以社区为场地, 其更注重实用性、民主性、开放性及其大众参与性, 强调居民的参与、根植于社区并试图用艺术的方式去 探讨问题。结合艺术家苏珊的理论与本土实践的探索, 社区公共艺术逐渐开始成为了城市微更新的新手段, 以艺术促进公众的参与, 构建人与人之间的关系, 美 化着城市的环境。

\section{3. 生态美学与社区公共艺术}

自 20 世纪后期以来,随着现代工业文明对自然的 破坏以及人对自身与自然关系的迷茫,生态问题逐渐 进入美学界研究视野,并成为美学主要研究对象之一。 天津大学王鹤教授认为自然环境公共艺术与生态美 学从根本上是同源的,是从不同方向朝一个终极目标 的努力,完全可以在理论与实践上互为补充并互相促 进, 并且自然环境公共艺术建设中的人类行为与生态 美学的研究对象并不抵触。特别是在城市更新中生态 和环境保护备受关注的今天，

公共艺术在介入城市和社区空间的实践中同样 注重生态问题。并且生态美学是生态学与美学的交叉 学科, 及用美学的视角去审视生态学或者以生态学的 视角去审视美学。公共艺术又是探讨美学问题和探讨 着社会问题, 因此在社区中关注生态, 探讨美学并根 植社区的公共艺术实践是及其有意义的。

\section{2. 社区基本情况分析}

\section{1. 环境研究分析}

\section{1.1 自然环境}

重庆地属热带季风性湿润气候, 年均气候在 18 摄氏度左右，冬暖夏热，无霜期长，雨量充沛，常年 降雨量在 1000-1450 毫升, 春夏之交夜雨尤甚, 因此 有 “巴山夜雨”之说, 地处巴蜀盆地, 重庆年平均相 对湿度多在 $70 \% \sim 80 \%$, 在中国属高湿区。并且重庆 是我国重要的中药材产区,其湿润的气候,复杂的地形 地貌,形成了重庆复杂多样的生态环境,有利于多种生 物共存, 同样也造就了重庆丰富的药用植物资源。

\subsection{2 历史人文}

铁路三村位于重庆九龙坡老工业区黄桷坪街道, 社区建于 20 世纪 70 年代末, 是成都铁路总局重庆 工务段下属的家属住区, 现如今社区的环境逐渐没落, 成为了老旧工业社区，但随着城市的发展社区内工人 的年轻子女更多选择搬向环境更好的新社区, 这也导 致社区人口老龄化趋势愈发的严重, 随着时间流失, 社区的文脉逐渐流失。

\section{2. 老旧社区公共艺术介入优势分析}

\section{2.1 社群关系}

与其他城市社区不同的是铁路三村内部的居民 基本都是铁路职工退休的工人或家属，居民彼此之间 相互了解。此外 2019 年四川美术学院师生团队在该 社区已经举办过一次公共艺术介入社区的活动, 社区 目前还保留一些公共艺术作品, 且反响很好, 居民对 于艺术介入到自己的生活已经非常接受。因此在社群 关系层面，居民与居民，居民和艺术家之间都已经建 立了良好的关系。

\subsection{2 社区资源}

首先社区拥有浓厚的铁路历史文化, 为公共艺术 设计提供了特色的创作元素。其次社区建筑延续着重 庆的山地特色使得社区空间存在高差, 形成了一些上 坡和下沉的空间，丰富了社区空间结构。最后依托于 重庆温暖湿润的气候社区内植物生长茂盛, 生态环境 优越, 在前期调研中发现社区存在着多种中草药资源。

\section{2 .3 审美水平}

铁路山村距离四川美术学院仅一公里以内, 周边 活跃着众多艺术家及优秀的艺术作品, 居民长期主动 或被动的接触到各种艺术作品, 无形的提高了居民的 审美水平，使得居民更好接受艺术。

\subsection{4 介入优势}

项目采用了 $1+1$ 的机制, 及居民与艺术家共创的 方式, 让居民参与并共同完成作品, 这样的形式能够 有效激发居民的参与度和积极性, 为作品最终的落地 提供了良好的支持。另外组织方四川美术学院邀请了 八所著名艺术和设计高校的研究生和教师参与, 专业 的团队介入使得居民更加放心。

\section{3. 作品选址分析}

在进行设计之间作者对社区空间结构和现有作 品情况以及社区人流聚集点进行分析，在设计之前提 前规避做出相似的作品, 且确保作品安置点位的合理。 2019 年秋季四川美术学院师生团队,已经进入社区进 行了一批在地创作，并有了一系列优秀的成果，在 
调研中笔者发现墙绘壁画类的作品偏多, 雕塑装置类 偏少。以火车为主题的创作偏多, 以社区其他元素展 开创作的作品较少。因此在调研后能够提前规避一些 创作类型。此外作者分析了社区内一天中人流量较大 的节点位置并结合现有作品的位置, 分析出了人流量 大且尚未改造的节点, 并选择出适合个人发挥且适合 公共艺术介入的空间节点作为创作点位 (图 1, 图 2)。

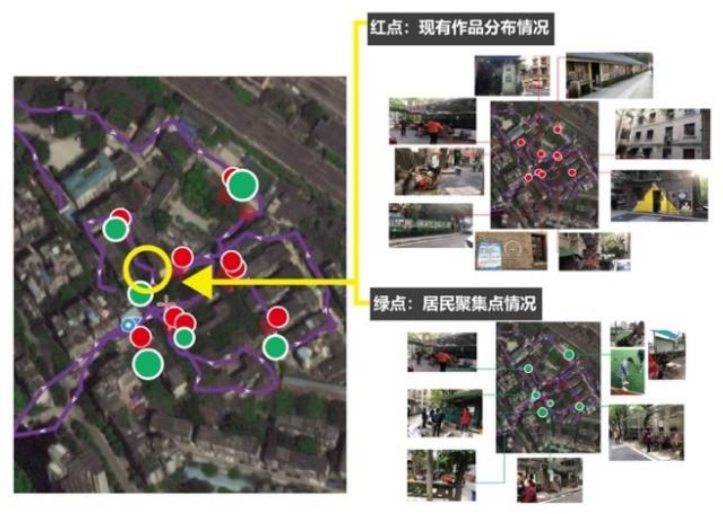

图 1 作品选地分析

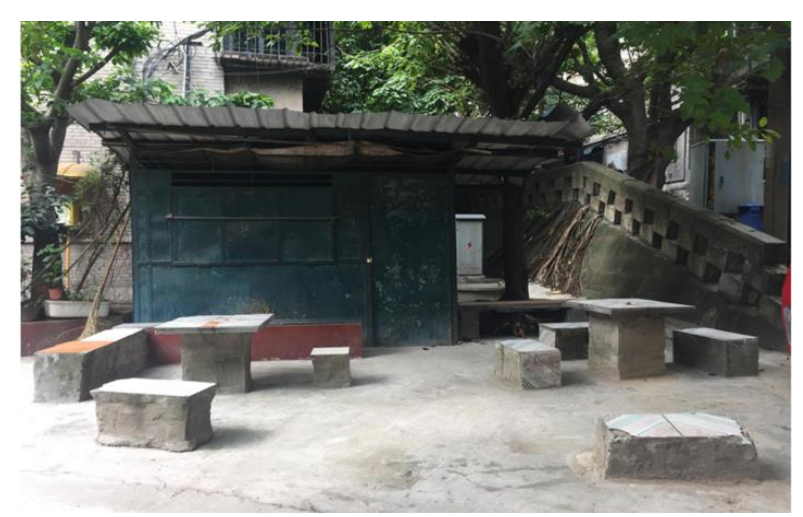

图 2 作品选地

\section{3. 生态美学下的社区公共艺术设计实践}

\section{1. 项目概况}

本项目是由四川美术学院组织发起的重庆生态 艺术季平行板块长江上下公共艺术行动下的一件社 区公共艺术设计作品。作者通过多方合作深入到社区 中观看和思考问题, 从生态美学的视角出发去设计公 共艺术作品, 并结合公共艺术的在地性、公共性、参 与性、生态性的原则, 创造出适合社区空间环境的公 共艺术作品, 从而提高社区空间环境的观赏性和艺术 性, 为社区居民提供交流场所。结合生态美学通过作 品引起观者对生态环境和对社区资源进行重新审视。

\section{2. 设计理念}

结合前期调研, 从暂无公共艺术作品和人流量较 大的空间节点进行选址, 在不破坏社区原有肌理和从 生态角度出发, 在材料上选择可持续性的或尽量使用 社区现有资源进行废物利用, 避免短期作品出现和资
源浪费。从人与作品的角度思考, 让作品与社区居民 产生互动, 吸引居民主动关注社区内 “消失的记忆”。 另外从空间控制考虑, 设法从消极空间入手改造成社 区积极空间。另外尽可能让作品满足功能需求, 避免 设计出纯观赏性的作品从高效利用社区资源, 维护居 民利益。

在调研中作者发现了社区草地中有几位中草药, 于是从中草药作为切入点, 通过与居民交流联系了对 中草药感兴趣的居民一起在社区内寻找更多的中草 药 (图 3), 下一步结合所选点位和作者构思展开公共 艺术的设计和策划。作者结合调研将绿皮铁房用艺术 的表现形式设计成中草药箱, 与药房中草药箱不同的 是社区中的草药箱里种植的是鲜活的中草药植物。将 这一概念和效果图向居民讲解, 询问居民们的意见并 邀请参与共创。(图 4)
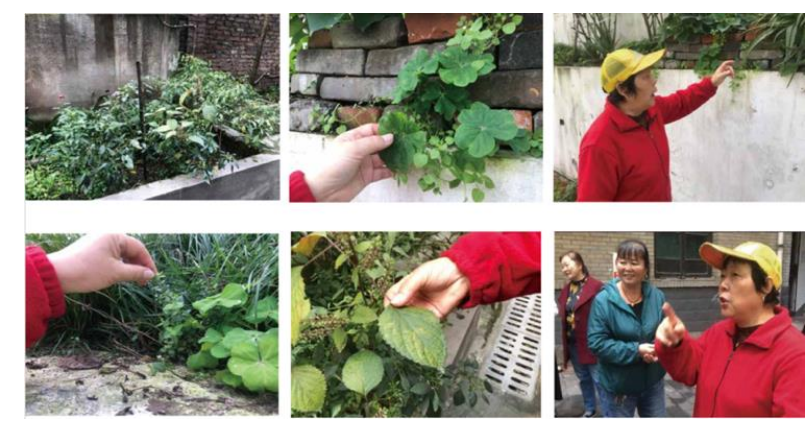

图 3 与居民共同寻找社区草药

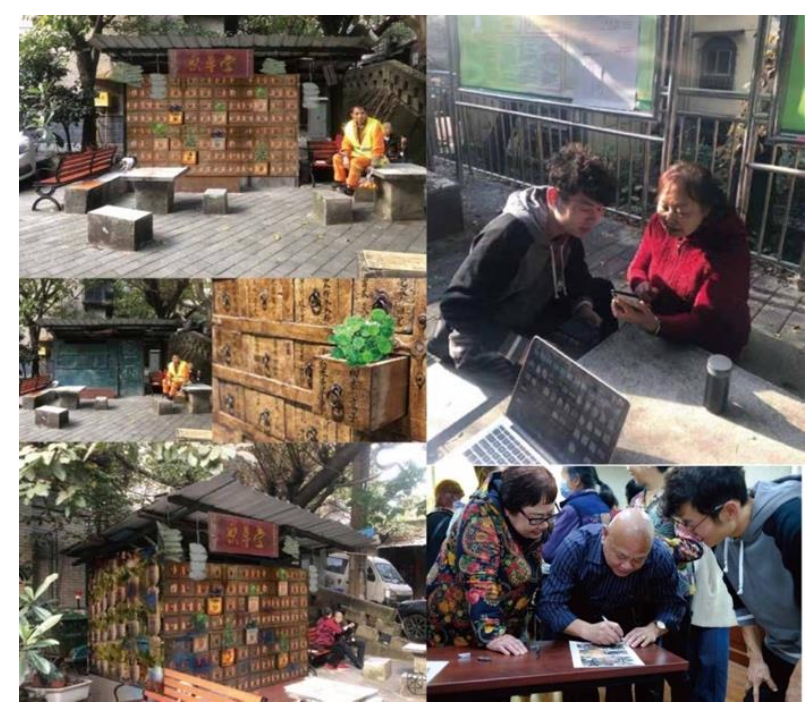

图 4 询问居民意见

\section{3. 公共艺术活动策划}

收集意见的同时也在吸引着对种植项目感兴趣 的居民, 打好建立种植社群的基础。此外作者计划举 行一场种植工作坊并提前邀请居民通过海报上的二 维码加入到微信社群中, 一方面加强居民与居民之间 的沟通交流, 另一方面为公共艺术活动招募更多志愿 者和参与者, 增加活动的影响力。作者将公共艺术活 动按照时间线分为以下 7 个步骤: 
(1)成立社群: 居民一直对种植感兴趣但是社区没 有人主动组织, 针对此问题作者策划种植活动, 招募 社区内对种植感兴趣的居民共同参与规划与种植, 促 进社区居民形成爱好种植的居民自组织。

(2)宣传海报: 设计宣传海报并粘贴在社区人流量 较大的节点, 吸引居民关注, 并通过海报上的二维码 加入社群，一方面方便后续通知居民参加工作坊，另 一方面可以扩大社区的规模, 让项目影响更多居民 (图 5)。
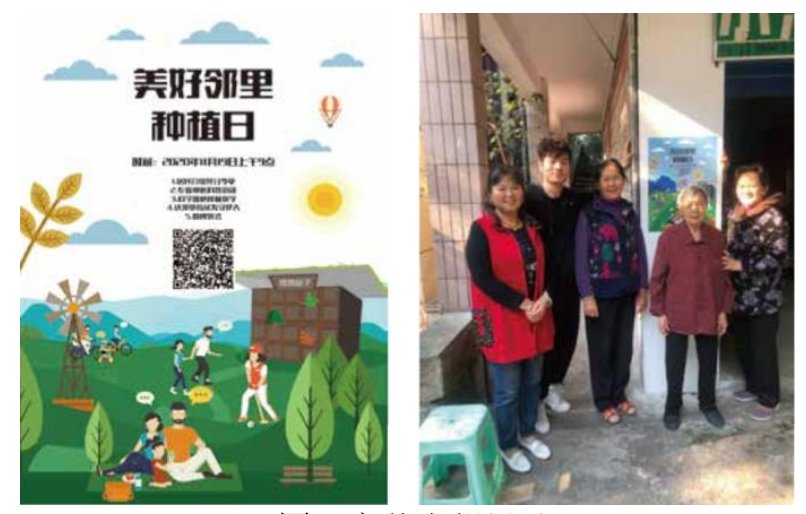

图 5 宣传海报设计

(3) 艺术改造: 邀请居民共同参与铁皮屋的清理 和绘制, 有难度的部分由作者完成, 易操作的部分与 居民共同完成，增加居民的参与度和对作品对认同感。 并从居民家中收集废旧塑料品, 带领居民将其改造成 彩绘的种植容器, 让废旧资源再利用, 并将周边桌椅 进行翻新, 与作品整体风格达到一致。

(4) 举办工作坊: 通过前期与居民关系的建立和 维护, 提前制定工作坊的日期, 让居民从自己家拿出 一株中草植物, 以个人的名义种植在自己制作的种植 容器中, 并与居民介绍植物的名字和功效, 加强邻里 之间的关系并发扬居民分享奉献的美德（图 6)。

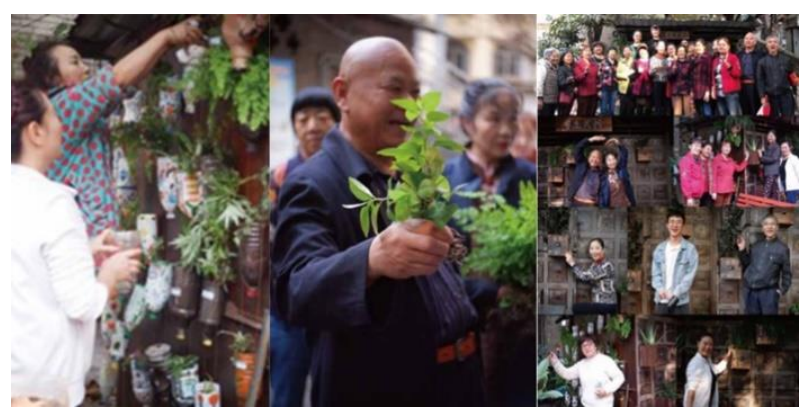

图 6 种植工作坊

(5) 制定维护机制: 制定草药种植和采摘规则, 制定 “守护人” 机制, 既邀请每户分享、认领并守护 一盆中草药进行长期维护, 让草药变成分享给居民的 社区公共资源。

(6)草药科普: 把收集到的草药信息进行整合, 并询问专业中医其功效和使用方法, 再进其进行归纳 整理成科普传单 (图 7), 分发给社区居民, 使社区居 民重新审视自己身边的资源和生态。

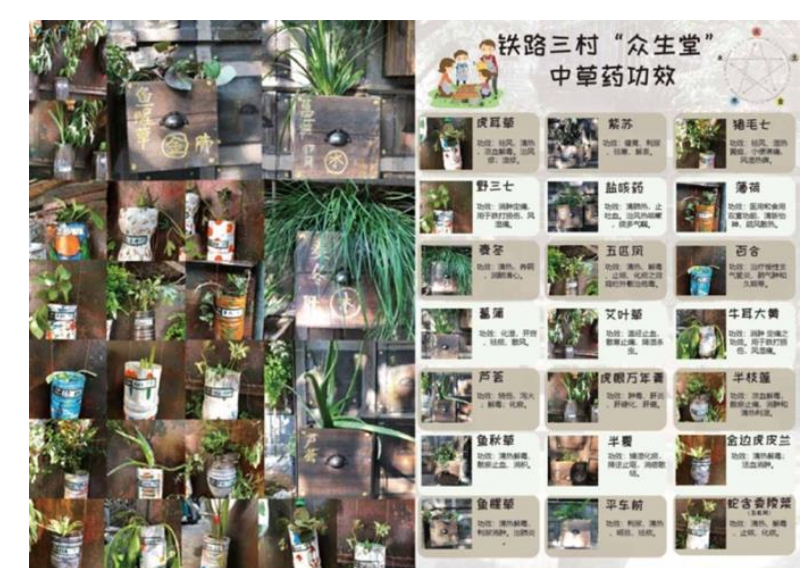

图 7 中草药功效宣传

(7) 维系社群: 邀请参与活动和社区内对种植感 兴趣的居民进入微信群，在群中分享与中草药、生态 相关的知识, 让居民更加了解社区环境的同时更能加 强中草药社群的凝聚力从而使得变得更加可持续。

\section{4. 设计说明}

作品《众生堂》为大小 3 米 $\times 2$ 米 $\times 1.8$ 米的公共 艺术装置, 以传统中草药铺为空间原型, 关注公众性 与在地性的需求, 从生态性出发创作可持续性发展的 社区公共空间。具体实施时, 通过公共艺术的介入, 将原先用来放置清洁用品的绿皮屋改造成铁路三村 共有的新鲜中草药铺, 并以废旧塑料瓶作为种植容器, 充分利用社区现有的中草药资源, 动员对种植感兴趣 的居民，激发居民共同参与草药种植的社区微更新活 动。这个可持续发展的社区中药铺将焕发勃勃的生机, 激励公众重新审视社区可利用的现有公共资源, 并设 置社区草药守护人的后期维护运行机制，让居民以个 人身份自觉维护属于自己的那份草药, 并带动和鼓励 他人将草药相互分享, 促进社区邻里交流, 共同创建 和谐美好社区（图 8)。

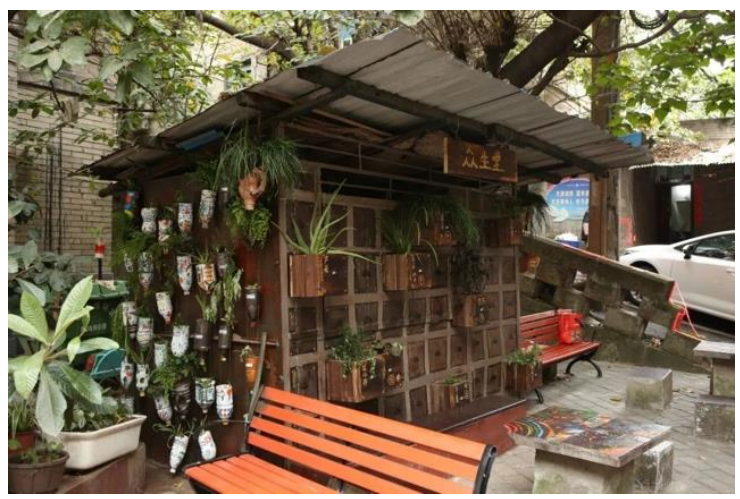

图 8 作品最终效果

\section{4. 经验总结}

在项目前期应扎根于社区进行在地调研, 发觉社 区资源, 在沟通交流的过程中与居民互相建立信任和 良好的关系, 从而使方案从概念到实施全过程都吸引 了居民主动参与, 使居民能通过艺术创作对自己的作 
品产生情感连接, 一定程度上能够激活对项目的认同 感, 形成对作品的主人翁意识, 更有利于作品的后期 维护。在作品关键节点的把控上由更专业的人去实施, 如草药箱的绘制和药盒的位置设定, 专业人员的介入 能保证作品的整体效果, 提高作品的品质, 而居民的 部分参与能提升作品的温度和加强作品的意义, 二者 结合能够使作品更加完整并使作品真正融入到居民 生活当中。种植日当天居民从自家带出一株中草药, 并种植在自己绘制的容器里, 写上自己的姓名成为这 株植物的守护人, 负责为自己守护的中草药浇水, 容 器里的植物是居民可以共享的, 居民以个人名义分享 给其他社区居民, 这种共享的模式有效的促进居民之 间的感情, 使社区更有凝聚力。作品完成后居民自发 组织取名并投票，最终选出 “众生堂” 作为这个草药 箱的名字, 也象征着居民共同合力完成作品后和睦共 处的和谐生机的愿景。

\section{5. 结语}

众生堂公共艺术作品的实践过程, 充分利用了社 区内现有的资源, 以生态美学为前提, 以公共艺术作 为触媒, 美化了社区公共空间的环境, 促进了社区居 民的沟通交流更增强了社区居民之间的凝聚力, 让生 态的公共艺术作品在社区有机且可持续的生长。居民 在通过参与共创的实践过程中重新审视了社区的资 源, 学会认识美, 创造美, 分享美的同时更提高了合 理利用利用和保护生态环境的意识。此项目改造过程 希望能为今后生态美学视角下的社区公共艺术设计 实践提供实践思路。并倡导艺术家在实践中既要做美 的艺术更要做生态的艺术, 在美化社区环境的同时, 更重要的是呼吁居民关注周边生态环境, 通过作品让 生态变得更美好。

\section{REFERENCES}

[1] Zeng Fanren. A Test of Ecological Aesthetics [J]. Literary and Art Studies, 2002(05):11-16.

[2] The new type of public art. [US] Susan Lacey. Lacey, Wu, Ma, Active. Far Stream Publishing Co. 1984

[3] Wang He. The application of ecological aesthetics in the creation and criticism of public art in natural environment $[\mathrm{J}]$. Journal of Central South University for Nationalities (Humanities and Social Sciences Edition), 2012,32(01):135-137. 